\title{
APRENDIZAJE CON BASE EN PROYECTOS PARA DESARROLLAR CAPACIDADES DE PROBLEMATIZACIÓN EN EDUCACION SUPERIOR
}

PROJECT-BASED LEARNING TO DEVELOP PROBLEMATIZATION SKILLS IN HIGHER EDUCATION

\section{Volumen 11, Número 1}

pp. 1-19

Este número se publicó el 30 de abril de 2011

\author{
Juan Manuel Muñoz Cano \\ Teresita del Niño Jesús Maldonado Salazar
}

La revista está indexada en los directorios:

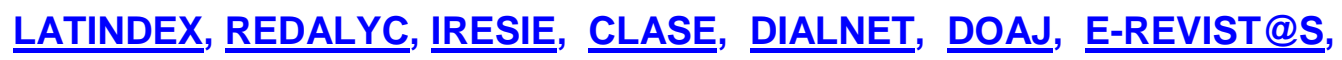

La revista está incluida en los sitios:

$\underline{\text { REDIE}}, \underline{\text { RINACE}}, \underline{\text { OEI }}$ MAESTROTECA, PREAL, $\underline{\text { HUASCARAN }} \underline{\text { CLASCO }}$ 


\title{
APRENDIZAJE CON BASE EN PROYECTOS PARA DESARROLLAR CAPACIDADES DE PROBLEMATIZACIÓN EN EDUCACION SUPERIOR \\ PROJECT-BASED LEARNING TO DEVELOP PROBLEMATIZATION SKILLS IN HIGHER EDUCATION
}

\author{
Juan Manuel Muñoz Cano' \\ Teresita del Niño Jesús Maldonado Salazar ${ }^{2}$
}

\begin{abstract}
Resumen: El propósito del estudio fue diseñar una intervención educativa para que los estudiantes superaran las dificultades en el manejo autónomo de la Internet, y que a través de este recurso desarrollaran capacidades para la identificación de los problemas de salud que afectan a la población, así como la búsqueda y análisis de información clínica actualizada. Se trabajó con base en proyectos y la solución de problemas durante cuatro meses con 31 estudiantes a quienes se asesoró en el manejo de bibliotecas virtuales. Se emplearon distintas estrategias en el aula para el trabajo en espacio virtual, como la elaboración del árbol de problemas y el árbol de soluciones. La evaluación formativa facilitó el seguimiento del proceso de aprendizaje y la sistematización de los resultados. La evaluación se apoyó en criterios definidos en matrices de evaluación y en el portafolio de evidencias. La acreditación se realizó con base en la calidad de sus informes. La experiencia la terminaron 14 estudiantes, y 12 de ellos lograron elaborar informes complejos que incluyeron conclusiones y recomendaciones y la consulta de varias fuentes localizadas en bibliotecas virtuales.
\end{abstract}

Palabras clave: EVALUACIÓN FORMATIVA, APRENDIZAJE AUTOREGULADO, AUTOMONITOREO DE APRENDIZAJE, PROBLEMATIZACIÓN

\begin{abstract}
The purpose of this study was to design an educational intervention for students to overcome the difficulties in the autonomous management of the Internet, and through this appeal will develop skills to identify health problems that affect people as well as search and updated clinical data analysis. We worked based learning projects and problem solving for four months with 31 students who were advised to use the virtual library. Different strategies were used in the classroom to work in virtual space, such as drafting the problem tree and the tree of solutions. Formative assessment facilitated the learning process monitoring and results systematization. The evaluation was based on criteria in rubrics and portfolio of evidence. The accreditation was made based on the quality of their reports. The experience was concluded 14 students, and 12 of them managed to produce complex reports that included findings and recommendations and consultation of various sources located in virtual libraries.
\end{abstract}

Key words: FORMATIVE ASSESSMENT, SELF-REGULATED LEARNING, SELF-MONITORING OF LEARNING, PROBLEMATISATION

\footnotetext{
${ }^{1}$ Especialista en Educación Superior, Maestro en ciencias en biomedicina molecular por el Instituto Politécnico Nacional, México. Profesor de fisiopatología y proyecto de investigación en la Escuela de Medicina de la Universidad Juárez Autónoma de Tabasco, México. Dirección electrónica juan.munoz@dacs.ujat.mx

${ }^{2}$ Maestra en Educación Ambiental por la Universidad Pedagógica Nacional, México. Profesora de Pedagogía en la Universidad Pedagógica Nacional, unidad 045, México. Dirección electrónica tmaldonado@semarnat.gob.mx
}

Artículo recibido: 21 de noviembre, 2010

Aprobado: 25 de abril, 2011 


\section{Introducción}

Con el fin de desplazar la pedagogía del ámbito del examen de tipo test como actividad central del proceso educativo -donde operan relaciones de poder más que de saber y una lucha entre el docente y el alumno por la calificación (Bernstein, 1998, p. 134; Díaz Barriga, 2009, p. 138)- hacia el ámbito de la problemática real que ellos atenderán a su egreso, hemos desarrollado y sistematizado experiencias pedagógicas hacia la formación metodológica con auxilio de las TIC (Muñoz, Maldonado y Córdova, 2010; Muñoz, 2011). Sin embargo, durante la puesta en práctica, hemos notado dificultades en el logro para la identificación de problemas, de sus causas, la interrelación de las consecuencias, y el sustento en la literatura especializada que se encuentra en línea. Esto se debe a que los estudiantes frecuentemente trabajan en las clases expositivas, donde se privilegia la memoria mecánica. Así, no se construyen capacidades para formar competencias profesionales (Agencia Nacional de Evaluación de la Calidad y Acreditación [ANECA], 2008, p. 75).

Con estas prácticas no se afronta, y mucho menos se resuelve la cuestión de los diferentes niveles culturales que tienen los estudiantes, ya que no se toman en consideración "sus imágenes familiares, el capital cultural que poseen, el medio en que se desenvuelven" (Díaz Barriga, 2009, p. 148). Por el contrario, el trabajo en situaciones complejas, a semejanza de la vida real, permite construir conceptos, sustentar aprendizajes prácticos y resolver problemas. La educación superior requiere de un proceso educativo innovador, de estrategias apropiadas para transitar a esos escenarios, pues "es evidente que en la mayoría de los casos la situación acaba pervirtiéndose hasta el extremo que los propios estudiantes piden recetas para solucionar problemas antes que aplicar su propio razonamiento para hacerlo" (Baños y Pérez, 2005).

\section{Marco conceptual}

El reto de la educación superior es formar cuadros profesionales que contribuyan en la transformación de la sociedad, lo que implica -entre otras cosas- resolver problemas desde filosóficos hasta médicos. El paradigma de la transmisión del conocimiento supone que se aprende de manera lineal, y por medio de la repetición, lo cual no tiene sustento. Se sabe que el aprendizaje es caótico, complejo, vinculado a las emociones y a la consciencia; se parte de las concepciones previas para construir nuevos conceptos (Picquart, 2008). Para resolver problemas no es suficiente con memorizar información. La idea general que la gente Volumen 11, Número 1, Año 2011, ISSN 1409-4703 
aprende de manera lineal, y por medio de la repetición, no tiene sustento. Por el contrario, en las teorías agrupadas como constructivismo existe una mayor aproximación a lo que se ha podido demostrar acerca de cómo funciona la mente. Estas investigaciones, desde diversos enfoques, sustentan la necesidad de organizar escenarios diferentes, experiencias novedosas, para que los estudiantes puedan aplicar lo que aprenden -no en forma de definiciones o paradigmas- para usar, en nuevos escenarios, lo que han aprendido.

El conocimiento socialmente significativo, o "conocimiento utilizable" (Committee on Developments in the Science of Learning [CDSL], 2000, p. 9) no es equivalente a la memorización de datos desvinculados entre sí. Las técnicas docentes que se emplean en muchas escuelas, donde el profesor determina el ritmo, los contenidos, la secuencia, y los mecanismos de evaluación, en los escenarios virtuales, tienen como resultado las mismas limitaciones educativas que en escenarios reales. Enseñar no es fácil (Picquart, 2008), es necesario transformar la práctica docente para que, con auxilio de las tecnologías de la comunicación e información (TIC), sea en escenarios reales o virtuales, se posibilite guiar e incrementar el aprendizaje.

En el caso de la educación médica, ésta tiene como propósito la formación de un profesional que puede resolver problemas del área de la salud, que debería poder modificar las tendencias en morbilidad y mortalidad. Para esto es necesario que aplique en su práctica cotidiana los resultados de la investigación clínica disponible en la red (Abreu e Infante, 2008). En las escuelas de medicina donde el proceso educativo se basa en la solución de situaciones de la vida real, se han elaborado estrategias para que sus estudiantes establezcan prácticas de búsqueda y aplicación de la información disponible en bibliotecas virtuales.

Esto implica un proceso educativo donde desaparecen las asignaturas tradicionales y se trabaja más con base en las necesidades conceptuales de una situación particular y concreta. Esto se logra con modelos innovadores de enseñanza que son al mismo tiempo sistemas de aprendizaje: con base en problemas, proyectos y casos (SEP, 2006). Los modelos son unidades didácticas integradas en concordancia con la definición actual de la didáctica: la ciencia de la organización de los procesos de aprendizaje. La construcción de estas unidades didácticas se basa en la teoría experiencial de Dewey, aunque la construcción teórica más importante del aprendizaje con base en proyectos precede a "los principios de aprendizaje y a la práctica" (Kolmos, 2004). 
Aunque en América Latina hay antecedentes de experiencias de aprendizaje con base en proyectos y en problemas, son escasas. En las instituciones de educación superior, en general, los planes de estudio están diseñados con base en asignaturas distribuidas en dos ciclos que en medicina son el básico y el clínico. Ese diseño curricular ha sido analizado desde diferentes perspectivas y los resultados expresan que la fragmentación en asignaturas y el énfasis en la memorización para la repetición en el examen de tipo test -que ni puede ser justo ni objetivo- invierte la relación pedagógica (Díaz Barriga, 2009, p. 148).

Por ello, el proceso educativo ha de sustentarse en una evaluación constante de los avances y retrocesos de los estudiantes, la evaluación formativa. Este concepto fue elaborado por Scriven en los sesenta. Se cuenta que dijo: "La evaluación formativa es cuando el cocinero prueba la sopa que está preparando. La evaluación sumativa es cuando el cliente prueba la sopa". El cocinero no evalúa la calidad de la sopa por medio de un test, prueba la sopa y corrige. El cocinero tampoco hace una parte de la sopa, la termina, pues el cliente la espera con todos sus elementos, y éste tampoco la evalúa por medio de un test. Díaz Barriga (2009, p. 150) dice que Scriven buscó que el estudiante no se jugara toda la cuestión de la calificación en el último día de clases.

La evaluación formativa tiene por objeto determinar el progreso del estudiante durante el proceso de enseñanza y de aprendizaje en relación con el contexto en el que se desempeñará al egresar de la escuela. A pesar de ello, se pueden cuantificar y cualificar avances, ya que con este tipo de evaluación se pretende: a) identificar los logros y deficiencias durante la actividad educativa, b) obtener evidencias parciales de la efectividad de los elementos que intervienen en el proceso de enseñanza y de aprendizaje. En este sentido:

El uso de la evaluación formadora frecuente ayuda a que el pensamiento de los estudiantes se haga visible para ellos mismos, para sus compañeros y para su profesor. Esto permite la retroalimentación que orienta la modificación y el refinamiento del pensamiento. Dado el objetivo de aprender con comprensión, las evaluaciones deben propiciar que los estudiantes construyan un saber, y no la mera habilidad de repetir datos o desempeñarse en destrezas aisladas. (CDSL, 2000, p. 67)

El encontrar soluciones a los problemas de salud depende de su identificación en el contexto social, económico y cultural y, para el logro de ello -durante el proceso escolar- se requiere enseñar a aprender a construir el objeto de estudio, a problematizar. Este objeto de 
estudio es el problema de los pacientes o los grupos humanos que el médico ha de resolver con ayuda de información relevante, oportuna, disponible y accesible, lo que convierte a la red en una fuente necesaria para información, aunque estas fuentes son volátiles, inciertas, complejas y ambiguas (volatile, uncertain, complex, ambiguos, características del VUCA World).

Por lo anterior, el proceso de evaluación formativa se constituyó en el eje longitudinal del proceso educativo, se realizó para retroalimentar a los estudiantes a fin de que superaran las dificultades para el desarrollo de las capacidades necesarias en la identificación de problemas como paso previo para su solución con el apoyo de las TIC. Las preguntas que guiaron esta investigación fueron: ¿cómo construir un ambiente de aula (real y virtual) centrado en el conocimiento para que los estudiantes puedan ser conscientes de sus avances y retroalimentarse?, ¿qué ayudas necesitan los estudiantes para construir conceptos complejos? y, ¿cómo acompañar a los estudiantes en un proceso tendiente a su autonomía y su formación como estudiantes permanentes?

\section{Metodología}

Se realizó un estudio de intervención educativa (Coll, 1993) del tipo de investigación acción participativa (Mahtani y Sanz, 2008). Una intervención educativa es un proceso amplio y complejo surgido desde los docentes y su reflexión de la práctica (acciones, relaciones y significaciones); busca detectar problemáticas, explicarlas y proponer alternativas innovadoras de transformación. De este modo, se trabajó con un grupo de 31 estudiantes de séptimo semestre de medicina de la Universidad Juárez Autónoma de Tabasco, situada en el sureste de México, de febrero a mayo de 2010, a partir de contenidos de la asignatura Proyecto de Investigación, curso que se desarrolló de enero a junio de 2010. Normalmente el curso es exclusivamente presencial, pero en el diseño se incluyeron actividades en línea con el fin de configurar un entorno de aprendizaje con apoyo de las TIC de acuerdo con la categoría D de Coll et al. (2008).

Como asignaturas precedentes habían cursado, entre las más acordes como sustento esperado del estudio de acuerdo al paradigma de la transmisión y la lineariedad: metodología de la investigación, lectura y redacción, herramientas de la computación, inglés, fisiopatología, epidemiología y salud pública. 


\subsection{Diseño de la investigación}

El curso se diseñó con base en proyectos (Thomas, 2000), del tipo "de problemática", pues la identificación de ésta dirige el proyecto (Kolmos, 2004). Ya que este tipo de intervenciones debe tener un producto (Helle et al., 2006), se decidió que éste sería un informe donde se detallara la identificación de una problemática de salud en la comunidad donde vivían los estudiantes, sus causas y el contexto en que se desarrollaba. El trabajo sistematizó el proceso de construcción de un texto basado en el análisis que los estudiantes hicieron de un problema de salud, con énfasis en el contexto sociocultural y las interrelaciones con otros problemas para elaborar conclusiones y recomendaciones. Las tareas se realizaron para fomentar la capacidad de problematización, entendida como la identificación tanto de las causas de los problemas de salud, locales y nacionales, como de las interrelaciones de éstos, sus orígenes y el diseño de estrategias de solución. Las tareas tenían como finalidad transversal el manejo de información en línea, la capacidad de argumentación, y el trabajo colaborativo.

\subsection{El programa de la asignatura}

Proyecto de investigación es una asignatura del plan de estudios de medicina de la Universidad Juárez Autónoma de Tabasco, en el sureste de México (UJAT). Tiene como definición operacional en el plan de estudios "capacidad de problematización". Esta se entiende como la identificación tanto de las causas de los problemas de salud, locales y nacionales, como de las interrelaciones de éstos, sus orígenes y las estrategias de solución.

\subsection{La estrategia didáctica}

La asignatura fue presencial con apoyos y actividades paralelas en línea. El diseño de las estrategias didácticas partió de la identificación de las capacidades de los estudiantes para buscar y manejar información y elaborar reportes con base en datos numéricos. Al principio de la etapa formativa se les proporcionó una guía didáctica que se colocó en la plataforma de la dependencia construida en Claroline disponible en www.dacsvirtual.ujat.mx.

La guía sustentó el trabajo con base en proyectos, e incluyó lineamientos para la búsqueda de información, sugerencias para ligas de consulta, elaboración de apuntes de las lecturas, elaboración de reportes y el uso correcto de las referencias y el respeto del derecho de autor. Como tarea se planteó la elaboración de proyectos acerca de problemas de salud, desde la perspectiva de causas y efectos con la finalidad de elaborar conclusiones y Volumen 11, Número 1, Año 201 1, ISSN 1409-4703 
recomendaciones aplicables directamente en su experiencia cotidiana. Los estudiantes debían enviar al correo electrónico del profesor sus avances, los cuales se les devolverían expeditamente una vez hechas las observaciones. Los contenidos conceptuales se dosificaron de manera que aumentara por etapas mensuales.

Tabla 1. Matriz de evaluación construida para el curso.

\begin{tabular}{|c|c|c|c|c|}
\hline Puntaje & 3 & 2 & 1 & 0 \\
\hline $\begin{array}{c}\text { Identificación del } \\
\text { problema }\end{array}$ & $\begin{array}{l}\text { Describe una } \\
\text { situación que } \\
\text { origina un problema } \\
\text { y sus } \\
\text { consecuencias }\end{array}$ & $\begin{array}{l}\text { Relaciona un } \\
\text { problema con alguna } \\
\text { de sus causas }\end{array}$ & $\begin{array}{l}\text { Menciona una } \\
\text { situación que es un } \\
\text { problema de salud }\end{array}$ & $\begin{array}{l}\text { Sólo menciona } \\
\text { temas sin } \\
\text { problematizar }\end{array}$ \\
\hline $\begin{array}{c}\text { Redacción de la } \\
\text { definición del } \\
\text { problema }\end{array}$ & $\begin{array}{l}\text { Elabora un escrito } \\
\text { fluido y sustentado } \\
\text { en datos } \\
\text { conceptuales y } \\
\text { numéricos }\end{array}$ & $\begin{array}{l}\text { Elabora un escrito } \\
\text { donde se pueden } \\
\text { identificar causas y } \\
\text { efectos del problema }\end{array}$ & $\begin{array}{l}\text { Elabora un escrito } \\
\text { donde no } \\
\text { interrelaciona los } \\
\text { conceptos para } \\
\text { apoyar la } \\
\text { argumentación }\end{array}$ & $\begin{array}{l}\text { Se limita a } \\
\text { transcribir } \\
\text { fragmentos de los } \\
\text { textos que } \\
\text { consulta. }\end{array}$ \\
\hline $\begin{array}{l}\text { Identificación del } \\
\text { dilema }\end{array}$ & $\begin{array}{l}\text { Identifica varias } \\
\text { posiciones, toma } \\
\text { una con respecto al } \\
\text { problema, sustenta } \\
\text { por qué no elige } \\
\text { otras }\end{array}$ & $\begin{array}{l}\text { Toma una posición } \\
\text { respecto del } \\
\text { problema pero no } \\
\text { identifica otras } \\
\text { facetas del mismo }\end{array}$ & $\begin{array}{l}\text { Elabora } \\
\text { definiciones } \\
\text { relacionadas con el } \\
\text { problema pero no } \\
\text { identifica diversas } \\
\text { posiciones }\end{array}$ & $\begin{array}{l}\text { Aborda el problema } \\
\text { con una visión } \\
\text { limitada que no } \\
\text { identifica } \\
\text { posiciones respecto } \\
\text { a éste }\end{array}$ \\
\hline $\begin{array}{c}\text { Manejo de } \\
\text { bibliotecas virtuales }\end{array}$ & $\begin{array}{l}\text { La información } \\
\text { proviene de fuentes } \\
\text { primarias o de } \\
\text { artículos y los cita } \\
\text { correctamente }\end{array}$ & $\begin{array}{l}\text { La información } \\
\text { proviene de fuentes } \\
\text { primarias o articulos } \\
\text { que la refieren pero } \\
\text { no las cita } \\
\text { correctamente }\end{array}$ & $\begin{array}{l}\text { Parte de su } \\
\text { información } \\
\text { proviene de fuentes } \\
\text { confiables }\end{array}$ & $\begin{array}{l}\text { Cita insuficiente de } \\
\text { artículos o de } \\
\text { páginas } \\
\text { electrónicas sin } \\
\text { calidad. }\end{array}$ \\
\hline $\begin{array}{l}\text { Elaboración de } \\
\text { conclusiones y } \\
\text { recomendaciones }\end{array}$ & $\begin{array}{l}\text { Sustenta las } \\
\text { conclusiones en el } \\
\text { análisis de la } \\
\text { información } \\
\text { consultada } \\
\end{array}$ & $\begin{array}{l}\text { Escribe enunciados } \\
\text { que no expresan } \\
\text { conclusiones y } \\
\text { recomendaciones } \\
\text { puntuales }\end{array}$ & $\begin{array}{l}\text { Enuncia ideas } \\
\text { inconexas } \\
\text { relacionados con el } \\
\text { problema }\end{array}$ & $\begin{array}{l}\text { Sus enunciados no } \\
\text { contienen } \\
\text { información } \\
\text { relevante respecto } \\
\text { al problema }\end{array}$ \\
\hline $\begin{array}{l}\text { Ortografía y } \\
\text { gramática }\end{array}$ & $\begin{array}{l}\text { Comete un máximo } \\
\text { de tres errores } \\
\text { ortográficos }\end{array}$ & $\begin{array}{l}\text { Comete un máximo } \\
\text { de diez errores } \\
\text { ortográficos }\end{array}$ & $\begin{array}{l}\text { Comete un error } \\
\text { ortográfico por línea }\end{array}$ & $\begin{array}{l}\text { No sigue las reglas } \\
\text { ortográficas }\end{array}$ \\
\hline
\end{tabular}

La primera, la identificación de un problema de salud, fue la elaboración de un concepto científico concreto como la prevalencia de un problema de salud con base en datos numéricos provenientes de fuentes confiables. La segunda requería mayor conocimiento del problema de salud como la identificación de una variable independiente y la definición de 
factores de riesgo. La tercera se basó en aplicaciones más complejas, tales como la identificación de dos o más variables independientes para la elaboración de conclusiones, base de recomendaciones para sus pacientes y el mismo estudiante.

En el cronograma, disponible en la guía del curso, se especificaron metas, fechas para entregas, así como mecanismos de apoyo, y una matriz de evaluación para que los estudiantes pudieran autoevaluar sus avances. No se pasó lista ni se hicieron exámenes, pues los avances en la elaboración de sus productos deberían ser las evidencias de aprendizaje. Como evaluación sumativa debería presentarse un informe de resultados con los mismos criterios de la matriz de evaluación, pero con los rubros antecedentes, problema, justificación de la investigación. Para la acreditación del curso se debía tener un $80 \%$ en sus avances y se ponderó de acuerdo a porcentajes. Baja cuando obtuvieron menos de $30 \%$ de puntos de la matriz de evaluación, media cuando obtuvieron entre $31 \%$ y $70 \%$, y alta de $71 \%$ y más. Las actividades se planearon de acuerdo con el cronograma que se encuentra en la guía y se propuso una matriz para evaluar los avances (Tabla 1). Al finalizar, se realizó una sesión donde los estudiantes evaluaron su aprendizaje y al curso. La acreditación se basó en la calidad de sus productos de investigación.

\section{Resultados}

\subsection{Estrategias previas de búsqueda en la red}

Los estudiantes no tenían mayor experiencia en identificar fuentes confiables de información o en jerarquizar los contenidos. La técnica que utilizaban era abrir Google $\otimes_{\text {, }}$ escribir palabras $u$ oraciones clave y leer lo que apareciera primero (90.6\%). Algunos buscaban en portales de información médica (6.25\%), uno empleaba "Preguntas de Yahoo!®”, y otro mencionó Medline Plus® como primera opción. Esta técnica de acceder a la primera liga que les mostraba el motor de búsqueda, que se convirtió en el primer obstáculo en la búsqueda de información, se explicó por varias razones. Los estudiantes eran conscientes de la gran cantidad de información; sin embargo, no sabían cómo podían discriminar lo que encontraban (65.25\%), ni de la existencia de portales y ligas de interés para su educación (9.3\%), y no tenían experiencia en leer artículos de medicina en inglés (9.3\%). Acerca de la sistematización de la información, sólo uno respondió identificar datos para un problema específico, pues su principal motivo para acceder a los motores de búsqueda era documentar los temas para exposiciones, o encontrar figuras para esas 
mismas exposiciones, pues sus profesores han delegado la función de explicar la clase a los alumnos.

\subsection{Manejo de la guía para realizar las tareas}

Con el fin de que se desplazaran por la plataforma, se elaboró una guía con instrucciones para identificar las distintas posibilidades en la plataforma. Los estudiantes no accedieron de manera inmediata. La dificultad fue la limitación que significó el acceso exclusivo en el campus, pues no hay internet inalámbrico para los estudiantes y en el centro de cómputo deben cubrir una cuota. Para resolver este problema se migró a una página que se había empleado con anterioridad, ya que excepto dos estudiantes, los demás tenían acceso a la Internet en sus domicilios. Los materiales se colocaron en: http://mx.groups.yahoo.com/group/fisiopatologia clinica/. A pesar de esto, los estudiantes no mostraron mayor conocimiento de los lineamientos generales de la guía, por lo que se discutió con ellos en el aula mediante una proyección, y se revisó con ellos los elementos de la página, para lo que se trabajó en un aula de docencia en el centro de cómputo.

\subsection{Identificación de un problema de salud}

Se solicitó a los estudiantes que identificaran una situación problema y la expusieran al resto de la clase. La primera respuesta se centró en un tema semejante a los que se encuentran en los índices de sus libros, por ejemplo, cáncer de mama, cáncer de cuello de útero, desnutrición. Se hizo énfasis en que deberían preguntarse las causas que hacen que una situación se convierta en un problema que hay que resolver. En esa semana, la elaboración careció de sustento, sobre todo de datos numéricos y de fuentes de información. Se discutió la necesidad de reflexionar por qué las cosas no son de otro modo, además de argumentar con base en información. Al término del plazo establecido, $45 \%$ de los estudiantes envió sus informes. Sin embargo, la primera elaboración fue la transcripción de páginas de la red, que algunos presentaban como "su trabajo", sin considerar derechos de autor.

\subsection{Exposición de sus intereses de investigación}

Se discutió con los estudiantes en las sesiones presenciales y $68 \%$ definieron problemas percibidos, aunque al principio desde la perspectiva de "temas", como los que 
exponen en sus otras asignaturas. Esto es resultado de que en su trayecto formativo no han adquirido la habilidad de estructurar un concepto con mayor profundidad a partir de distintas fuentes, más de una asignatura. Asimismo, se discutió con ellos de acuerdo con el esquema de los límites y contextos de los problemas, que no son los de las asignaturas o sus libros escolares, para elaborar la pregunta de investigación. Después de discutir acerca de causas y efectos -para que posteriormente elaboraran intervenciones terapéuticos o de prevenciónse logró una mayor aproximación, pues comenzaron a elaborar preguntas que debían ser resueltas con una investigación. En este punto se promovió que los estudiantes interrelacionaran lo que deberían aprender con lo que ellos sabían, sus conocimientos previos, ya que se hizo énfasis en preguntar qué preconcepto había elaborado el estudiante y la diferencia con una construcción de la literatura científica, además de que se realizó de manera colaborativa (Figura 1). Este giro se reflejó en sus informes, ya que enviaron escritos mejor estructurados.

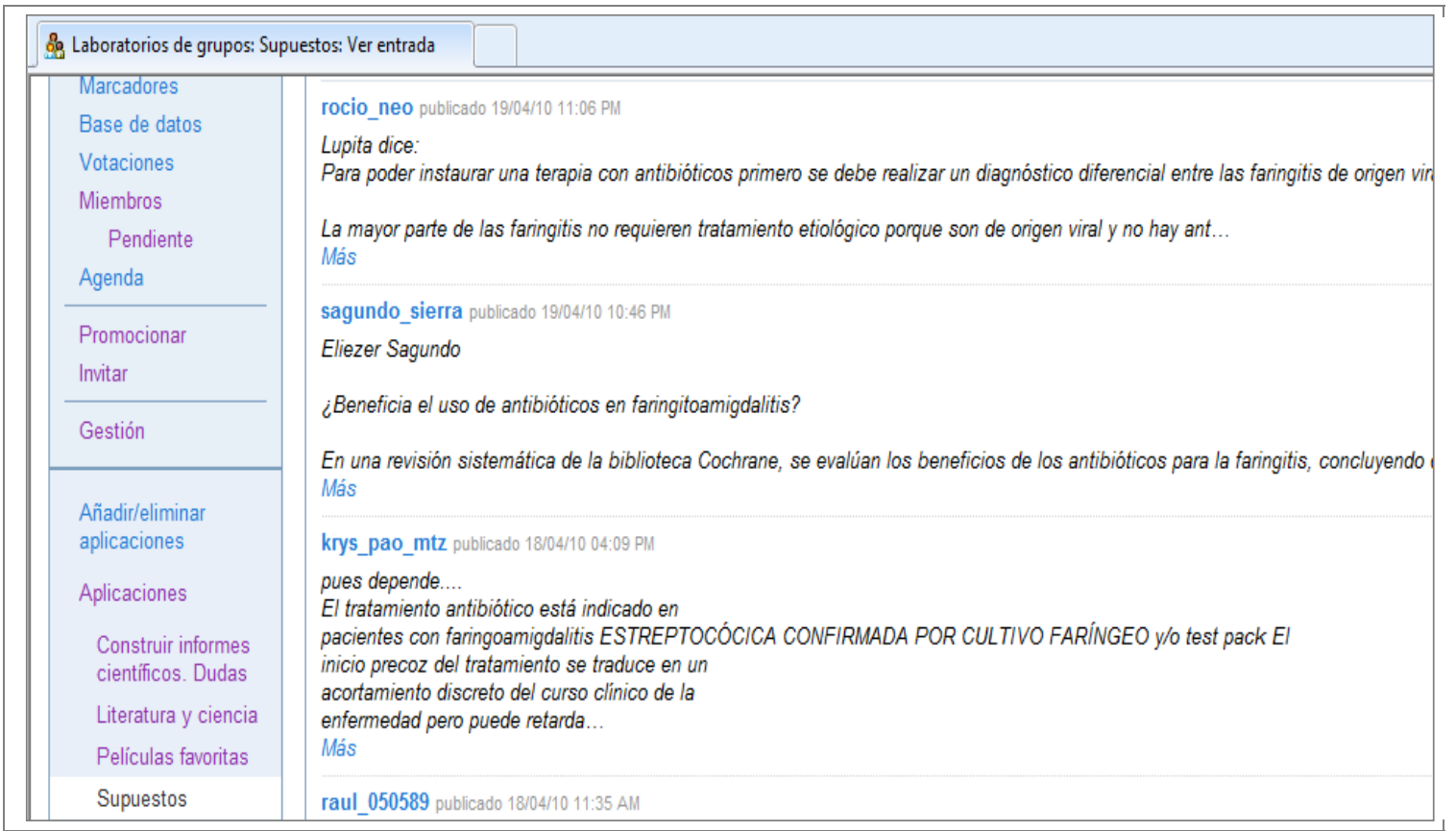

Figura 1. Pantalla de una discusión grupal acerca del uso y abuso de antibióticos. 


\subsection{Construcción del marco conceptual}

Se solicitó a los estudiantes que definieran una secuencia de pasos para construir el problema, los estudiantes mencionaron la elaboración de cuestionarios para responder a una pregunta de interés. Esta elaboración de cuestionarios es la idea que ellos tenían de hacer un proyecto de investigación, preguntas del tipo “¿qué sabe usted de...?”. Se trabajó con ellos para analizar las características de la lectura, las fuentes, la elaboración del escrito y su sustento, para lo que se revisó la guía en el aula, aunque ésta se encontraba disponible en la plataforma web. Una vez determinado que deberían sustentar sus problemas con datos numéricos, $51 \%$ lo hizo, y $45 \%$ reconstruyeron su delimitación del problema. Durante tres semanas más, $20 \%$ persistió en presentar transcripciones, hasta que lograron integrar las lecturas en un trabajo de mayor fluidez.

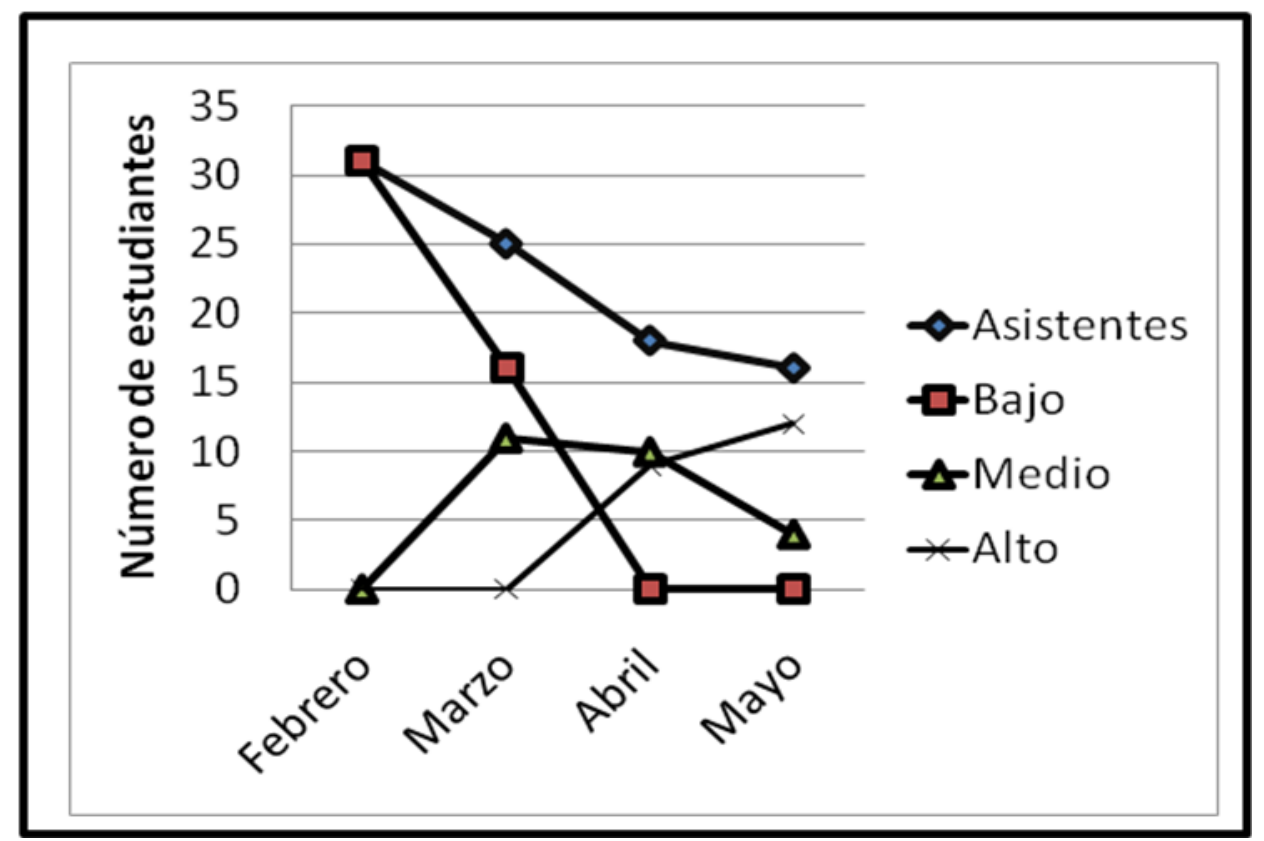

Figura 2. Ponderación de los resultados de los estudiantes de acuerdo a la matriz de evaluación.

En este punto se trabajó con la elaboración del árbol de problemas y el árbol de soluciones. Esta imagen gráfica permitió explicar las conexiones entre un fenómeno y sus consecuencias y entre las consecuencias y una intervención para modificar esa causa. Esta estrategia permitió agilizar la modificación de ideas iniciales. Al finalizar esta etapa, dos terceras partes del curso, $28 \%$ presentó su informe en tiempo, aunque $11 \%$ no cumplía Volumen 11, Número 1, Año 2011, ISSN 1409-4703 
completamente los criterios de la matriz de evaluación. Los estudiantes lograron elaborar preguntas de investigación, ejes para la búsqueda de información y datos.

Con el fin de buscar soluciones a este problema se elaboraron guías; sin embargo, la estrategia que dio mejores resultados fue trabajar en un aula equipada con un pizarrón electrónico y computadoras para los estudiantes ya que, aunque saben emplear la Internet, lo hacían sólo mediante motores de búsqueda y no en bibliotecas virtuales. Al final de la experiencia se logró que $17 \%$ de los estudiantes elaboraran reportes con referencias correctamente elaboradas, algunas de ellas en inglés, idioma del que no tienen dominio. Estos estudiantes elaboraron conclusiones e identificaron los puntos donde requerían de recomendaciones para los pacientes, incluso en el nivel personal y sus propias familias (Figura 2).

\section{Discusión}

Las dificultades para que los estudiantes construyan proyectos y aprendan a investigar se agrupan en tres rubros: a) las relacionadas con el grado de autonomía, b) las relacionadas con los conceptos, c) las relacionadas con los procedimientos.

\subsection{Dificultades relacionadas con el grado de autonomía}

Ya que para la resolución de problemas es necesario aprender a identificarlos, se sistematizó el resultado de una intervención educativa con estudiantes de medicina. Al principio los estudiantes mostraron escaso manejo de la información en línea y apego al método expositivo, la memorización y los exámenes, así como poca disposición para el trabajo escolar en línea, ya que de 31 estudiantes inscritos sólo 14 terminaron esta experiencia. También fue evidente la poca disposición inicial para afrontar tareas diferentes de preparar diapositivas de PowerPoint ${ }^{\circledR}$ o memorizar para un examen, pues sólo 11\% terminó en tiempo la elaboración de un primer informe.

Esto también se hizo evidente en que los estudiantes no manejaron como estrategias de autoevaluación sus propios avances, para guiarlos se les proporcionaron matrices de evaluación. No fue sino hasta finalizar el curso que los estudiantes se atrevieron a manifestar sus sentimientos con respecto al trabajo en el aula y en línea, sus dificultades y la frustración que significaba rehacer el trabajo. Esa supeditación de la escuela al poder, se configura en torno a la reproducción de la ideología -como deformación de la teoría- y que "es el modo de establecer relaciones" (Bernstein, 1998 p. 133). El sesgo de la conciencia de docentes y Volumen 11, Número 1, Año 2011, ISSN 1409-4703 
estudiantes es lo que hace que pocos tengan idea de lo que la escuela tiene como propósito, no en la forma de trabajo en el aula, sino en el aspecto de qué cambios deberá mostrar el estudiante al terminar el curriculum. Por ejemplo, Wang et al. (2007) se refieren a la evaluación formativa como los apoyos que necesita el estudiante para resolver las preguntas de un test. Precisamente porque los estudiantes se limitan a aprender para el examen, el estudiante no sabe qué espera de la escuela, qué es lo que podría demandar a la escuela para su formación, porque tampoco los docentes tienen claridad en el proceso global. Los docentes están preocupados por los contenidos de sus asignaturas, del manejo de sus códigos y de la división del trabajo con base en áreas de las asignaturas.

\subsection{Dificultades relacionadas con los conceptos}

¿Cómo ayudar a los estudiantes a ser autónomos? En este trabajo optamos por ayudar a los estudiantes a construir sus propios conceptos porque memorizar y repetir, o aprender paradigmas -los estudiantes de medicina consideran irrelevantes los cursos de asignaturas canónicas como anatomía y bioquímica (Watmough, O’Sullivan y Taylor, 2010)- no los prepara para la vida real. Aunque en los procesos educativos cada vez hay más evaluaciones iniciales, diagnósticas, en la práctica no es suficiente con esa aproximación, es necesario mantener un proceso continuo de acompañamiento para evaluar cómo los estudiantes aprenden.

Hay mucha evidencia de que el aprendizaje se incrementa cuando los maestros prestan atención a los saberes y creencias con que los aprendices llegan a una tarea de aprendizaje; usan estos conocimientos como punto de partida para la instrucción nueva y hacen un seguimiento constante de las concepciones cambiantes de los estudiantes a medida que avanza la instrucción. (CDSL, 2000, p. 7)

Conceptos como "formative summative assessment" de Wininger (2005), en realidad prácticas para responder mejor los exámenes, muestran la confusión conceptual existente en el campo, que construye definiciones para diferenciar entre "assessment" and "evaluation" (Dunn y Mulvenon, 2007), en el intento de mantener la validez del examen de tipo test. Responder mejor a las preguntas mediante la retroalimentación, a diferencia de lo que afirman Wang, Wangz, Wangz y Huang (2007), no significa comprender. Es precisamente esa posición lo que hizo que 15 de 31 estudiantes, acostumbrados a aprender para el examen, abandonaran esta experiencia. El propósito de que los estudiantes puedan ser 
independientes y manejarse solos, nos llevó a elaborar procedimientos y estrategias para aprendizaje metodológico acerca de construir textos tras la comprensión de conceptos, así como de la retroalimentación individualizada empleando el correo electrónico. Esta asesoría continua no puede aún sustituirse con un software para hacer exámenes, ya que responder preguntas para tipo test, como en la experiencia de Shah, Rajgor, Vaghasia, Phadtare, Pradhan et al. (2010), dista mucho de un verdadero proceso de escritura de artículos científicos. La escritura es una habilidad de alto nivel, y como tal, ha de desarrollarse a través de una instrucción sistemática y práctica (Silvia, 2007 p. 3).

\subsection{Dificultades con los procedimientos}

La falta de habilidades para la búsqueda de información es un problema definido en la World Alliance for Patient Safety (WHO, 2008). Hay estudios que demuestran que los médicos tenemos pocas habilidades para la búsqueda de información relevante y pertinente, que debe ser afrontado en la escuela (Davis, Paul, Mazmanian, Fordis, Van Harrison et al., 2007), lo cual es consistente con los hallazgos con los estudiantes que participaron en esta intervención. A semejanza de los estudios estos estudiantes han estado en contacto con las TIC y tienen habilidades en el manejo de los motores de búsqueda. Eso no significa el uso eficiente de la información, pues en el Reino Unido se documentó 89\% para motores de búsqueda contra $2 \%$ para las bibliotecas virtuales, así como la práctica de copiado y pegado, aunque los estudiantes reconocen la existencia de los derechos de autor (Rowlands, Nicholas, Huntington, Gunter, Withney et al., 2008).

A diferencia de los trabajos hacia el aprovechamiento escolar en ciclos, retroalimentamos a los estudiantes hacia prácticas más complejas como la medicina basada en evidencia (Straus, Green, Bell, Badgett, Davis et al., 2004). Trabajar para el examen limita la capacidad de asombro, la curiosidad, la posibilidad de afrontar problemas futuros. La respuesta está en modificar la forma de trabajo escolar. "La medición de la profundidad de comprensión puede presentarle retos a la objetividad. Hace falta trabajar mucho para minimizar el conflicto entre evaluar profundidad y evaluar objetivamente" (CDSL, 2000 p 9). A pesar de que sólo 16 estudiantes terminaron el curso, la respuesta al aprendizaje de uso de las TIC se ejemplifica en la respuesta de un estudiante, en la última sesión de clases.

Esta asignatura me ha brindado las herramientas necesarias para mejorar o modificar mis hábitos de estudio que tenía al entrar en la carrera. Es necesario saber que como médico estoy obligado a desarrollar métodos de aprendizaje basados en problemas 
para crear un conocimiento sustentado en bases científicas. Para ello es necesario darle la importancia debida al uso que le podemos dar al Internet y a otras fuentes de información para sacarle el máximo provecho. (C1, 27 de mayo del 2010)

\section{Conclusiones}

La práctica escolar donde las asignaturas se cursan con una secuencia predetermida a partir del supuesto de que esto facilitará la comprensión- debe transformarse para que el estudiante elabore su trayecto formativo de acuerdo con sus intereses y necesidades. Para que se produzca un aprendizaje significativo es necesario que se le dé valor social al contenido. Por otra parte, el modelo centrado en la exposición y la memorización para las preguntas del examen, hace que los contenidos sólo tengan valor para esta situación y se olviden rápidamente al día siguiente. Incluso en las situaciones en que se realizan reforzamientos, o repasos del material, ya que no tiene aplicación inmediata, se olvida tantas veces se memorice de nuevo.

El método del aprendizaje, con base en proyectos, puede relacionar el aprendizaje al contexto, ya que integra información y datos para analizar una problemática. También permite que los estudiantes construyan conceptos de manera colaborativa; sin embargo, este trabajo escolar de mayor complejidad es un obstáculo para los estudiantes acostumbrados a la pasividad a la que pareciera condenarles la escuela. Esto los lleva al abandono de la experiencia por preferir el sistema tradicional al que están acostumbrados y por tanto les parece más fácil aún cuando no construyan aprendizajes significativos.

La intervención educativa debe poner énfasis en la formulación de preguntas en vez de respuestas. Además, puede relacionar el contenido de aprendizaje con el contexto, lo que facilita la motivación y la comprensión del estudiante.

Es importante motivar a los estudiantes para apoyarse en las TIC como herramientas para el aprendizaje permanente. A pesar de que se piensa que los estudiantes tienen dominio de las TIC, en la práctica usan este recurso en forma limitada. Es necesario que los docentes formen capacidades necesarias en el manejo de lo que se encuentra en la red como elementos de aprendizaje, desde esquemas, videos, bibliotecas virtuales, hasta el uso de plataformas y cursos virtuales. De otra manera no es posible acompañar a los estudiantes en el proceso de aprender a aprender para que se conviertan en estudiantes permanentes. 
Se debe trabajar de manera constante para que los estudiantes comprendan que apoyar su práctica en conocimientos científicos no se limita a la memorización, sino que necesita del conocimiento y la aplicación de los avances publicados y disponibles en bibliotecas virtuales, así como en forma de normas oficiales y guías clínicas. Es importante acompañar a los estudiantes en la construcción de sus estrategias de aprendizaje permanente con apoyo en las TIC.

Es necesario que los estudiantes tomen conciencia de que deben responsabilizarse de su proceso de aprendizaje y tener como referente constante su práctica profesional, pues en la vida real no existe la clasificación de los conceptos como en las asignaturas escolares. En México se atribuye relevancia de los cursos de ciencia porque pretenden servir para la preparación de los estudiantes para entender los cursos de clínica -¿cómo, si los profesores de ciencia no tienen idea de lo que es la clínica?- y no con la finalidad de "conseguir una alfabetización científica para ejercer mejor la ciudadanía en un mundo cada vez más impregnado de ciencia y tecnología" (Acebedo, 2004). Para esto será necesario trabajar en el proceso de resolver problemas, más que presentar temas.

El modelo de enseñanza de "la racionalidad técnica", instaurado a finales del siglo XIX, plantea que el ejercicio de las profesiones "se reduce a aplicar reglas de carácter general deducidas de la ciencia o la tecnología. Se postula que la tarea clínica se limita a seleccionar la técnica correspondiente, $\mathrm{y}$, en consecuencia, las ciencias básicas tienen preeminencia sobre la clínica". Por lo tanto, "a la luz del conocimiento moderno la idea que interpreta a la práctica clínica como una derivación directa de las ciencias básicas", que, por lo tanto, éstas deben ser aprendidas antes de la clínica, "es insostenible" (Abreu e Infante, 2004).

\section{Referencias}

Abreu Hernández, Luis Felipe e Infante Castañeda, Claudia B. (2004). La educación médica frente a los retos de la sociedad del conocimiento. Gac Med Méx, 140 (4), 381-390. Recuperado el 20 de marzo de 2011 de: http://www.medigraphic.com/espanol/ehtms/e-gaceta/e-gm2004/e-gm04-4/em-gm044c.htm

Abreu Hernández, Luis Felipe; Cid García, Ángel; Herrera Correa Gloria, Lara Vélez Víctor, Laviada, Rocío y Sánchez Aparicio, Juan Antelmo. (2008). Perfil por Competencias del Médico General Mexicano AMFEM. Recuperado el 24 de marzo de 2011 de: http://www.amfem.edu.mx/intranet/descargas/competencias.pdf 
Acevedo Díaz, José Antonio. (2004). Reflexiones sobre las finalidades de la enseñanza de la ciencia: educación científica para la ciudadanía. Revista Eureka sobre enseñanza y divulgación de las ciencias, 1 (1), 3-16. Recuperado el 20 de marzo de 2011: http://www.apac-

eureka.org/revista/Volumen1/Numero 1 1/Educa cient ciudadania.pdf

Agencia Nacional de Evaluación de la Calidad y Acreditación. (2008). El profesional flexible en la Sociedad del Conocimiento. Informe ejecutivo. Recuperado el 20 de marzo de 2011 de: http://www.aneca.es/media/158162/informeejecutivoaneca jornadasreflexv20.pdf

Baños, Josep; Pérez, Jorge. (2005). Cómo fomentar las competencias transversales en los estudios de Ciencias de la Salud: una propuesta de actividades. Educ Med, 8 (4), 216225. Recuperado el 20 de marzo de 2011 de: http://scielo.isciii.es/scielo.php?pid=S1575-18132005000500006\&script=sci arttext

Bernstein, Basil. (1998). Códigos e investigación. Capítulo VI. En: Pedagogía, control simbólico e identidad (pp. 131-140). Madrid: Morata.

Centre for Educational Research and Innovation. (2005). Formative assessment: improving learning in secondary classrooms. OECD, Paris.

Coll Salvador, César. (1993). Constructivismo e intervención educativa: ¿cómo enseñar lo que se ha de construir? Propuesta Educativa, 8 (5), 48-57.

Coll Salvador, César; Mauri, M. Teresa y Onrubia, Javier. (2008). Análisis de los usos reales de las TIC en contextos educativos formales: una aproximación sociocultural. Revista Electrónica de Investigación Educativa, 10 (1), 1-18. Recuperado el 22 de marzo del 2011 de: http://redie.uabc.mx/vol10no1/contenido-coll2.html

Committee on Developments in the Science of Learning with additional material from the Committee on Learning Research and Educational Practice, National Research Council. (2000). How People Learn: Brain, Mind, Experience, and School. Expanded Edition. National Academy Press, Washington, D.C. Recuperado el 20 de marzo de 2011 de: http://www.nap.edu/catalog.php?record id=9853

Davis, David; Mazmanian, Paul E.; Fordis, Michael; Van Harrison, R; Thorpe, Kevin E. y Perrier, Laure. (2006). Accuracy of physician self-assessment compared with observed measures of competence. A systematic review. JAMA, 296(9):1094-1102. Recuperado el 21 de marzo de 2011 de: http://jama.ama-assn.org/cgi/content/abstract/296/9/1094

Díaz Barriga, Ángel Rogelio. (2009). El docente y los programas escolares. Los institucional y lo didáctico. México: IISUE.

Dunn, Karee E y Mulvenon, Aean W. (2009). A Critical Review of Research on Formative Assessment: The Limited Scientific Evidence of the Impact of Formative Assessment in Education. Practical Assessment, Research \& Evaluation, 14 (7), 1-11. Recuperado el 23 de marzo de 2011 de: http://pareonline.net/pdf/v14n7.pdf 
Helle Laura, Tynjälä Päivi y Olkinoura Erkki. (2006). Project-based learning in post-secondary education - theory, practice and rubber sling shots. Higher Education, 51, 287-314. Recuperado el 19 de marzo del 2011 de: http://www.springerlink.com/content/q16kw87373382451/

Kolmos, Anette. (2004): Estrategias para desarrollar curriculos basados a la formulación de problemas y organizados en base a proyectos. Educar 33, 77-96. Recuperado el 19 de marzo de 2011 de: http://dialnet.unirioja.es/servlet/articulo?codigo=989396

Mahtani Chugani, V.; Sanz Álvarez E. (2008). La evaluación cualitativa en la formación médica continuada. Educ Med, 11 (2), 77-83. Recuperado el 21 de marzo del 2011 de http://scielo.isciii.es/pdf/edu/v11n2/revision.pdf

Muñoz Cano, Juan Manuel, Maldonado Salazar, Teresita del Niño Jesús y Córdova Hernández, J.A. (2009). Formación para aprendizaje en línea de asignaturas de médico cirujano. Horizonte Sanitario 8, (2), 19-27. Recuperado el 20 de marzo del 2011

de: http://www.publicaciones.ujat.mx/publicaciones/horizonte sanitario/ediciones/2009 ma yo-agosto/03-Formacion aprendizaje.pdf

Muñoz Cano, Juan Manuel. (2011). Experiencia metacompleja para la construcción de un modelo de diagnóstico médico por estudiantes. Educ Med, 14. En prensa.

Picquart, Michael. (2008). ¿Qué podemos hacer para lograr un aprendizaje significativo de la física? Lat Am J Phys Educ, 2 (1), 29-36. Recuperado el 22 de marzo de 2011 de: http://www.journal.lapen.org.mx/jan08/LAJPE-Vol-2-No-1-2008.pdf\#page=32

Rowlands, Ian; Nicholas, David; Huntington, Paul; Gunter, Barrie; Withey, Richard; Dobrowolski, Tom; Tenopir, Carol; Williams, Pete; Fieldhouse, Maggie y Jamali, Hamid. (2007). Behaviour of the Researcher of the Future. University College London. Recuperado el 20 de marzo de 2011 de: http://www.publishing.ucl.ac.uk/behaviour.html

Secretaría de Educación. Subsecretaría de Educación Básica. Plan de estudios. (2006). Educación básica. Secundaria. Recuperado el 23 de marzo de 2011 de: http://www.reformasecundaria.sep.gob.mx/ciencia tecnologia/doctos/PLAN.pdf

Shah, Jatin; Rajgor, Dimple; Vaghasia, Meenakshi; Phadtare, Amruta; Pradhan, Shreyasee; Carvalho, Elias y Pietrobon, Ricardo. (2010). WriteSim TCExam - An open source text simulation environment for training novice researchers in scientific writing. BMC Medical Education, 10, 39. Recuperado el 23 de marzo de 2011 de: http://www.biomedcentral.com/content/pdf/1472-6920-10-39.pdf

Silvia, Paul J. (2007). How to write a lot: A practical guide to productive academic writing. Washington: Academic Pychologycal Association.

Straus, Sharon E; Green, Michael L; Bell, Douglas S; Badgett, Robert; Davis, Dave; Gerrity, Martha; Ortiz, Eduardo; Shaneyfelt, Terrence M; Whelan, Chad y Mangrulkar, Rajesh; for the Society of General Internal Medicine Evidence-Based Medicine Task Force. 
(2004). Evaluating the teaching of evidence based medicine: conceptual framework. BMJ, 329, 1029-1032.

Thomas, John W. (2000). A review of research on project-based learning. Texto en Internet. Recuperado el 23 de marzo de 2011 de: http://www.bobpearlman.org/BestPractices/PBL Research.pdf

United Nations Educational, Scientific and Cultural Organization. (2009). Quality assurance in Higher Education. Recuperado el 23 de marzo de 2011 de: http://www.iiep.unesco.org/en/focus-on-higher-education/quality-assurance-in-highereducation.html

Wang, Kuo-Hua; Wang, Tzu-Hua; Wang, Wei-Lung y Huang, Shih-Chieh. (2006). Learning styles and formative assessment strategy: enhancing student achievement in Webbased learning. Journal of Computer Assisted Learning, 22, 207-217. Recuperado el 23 de marzo de 2011 de: http://www.fi.uu.nl/ christianb/downloads/180509/wangetal2006.pdf

Watmough, Simon; O'Sullivan, Helen y Taylor, David. (2009). Graduates from a traditional medical curriculum evaluate the effectiveness of their medical curriculum through interviews. BMC Medical Education, 9, 64. Recuperado el 22 de marzo de 2011 de: http://www.biomedcentral.com/1472-6920/9/64

World Health Organization - WHO. (2008). World Alliance for Patient Safety. Forward programme 2008-2009. Paris: WHO. Recuperado el 22 de marzo de 2011 de: http://www.who.int/patientsafety/information centre/reports/Alliance Forward Program me 2008.pdf

Wininger, Steven R. (2005). Using your tests to teach: Formative summative assessment. Teaching Psychology, 32 (2), 164-166. 